\title{
Hyperfractionated Accelerated Reirradiation for Rectal Cancer: An Analysis of Outcomes and Toxicity
}

Randa Tao, ${ }^{1,2^{*}}$ C. Jillian Tsai, ${ }^{1,3}$ Garrett Jensen, ${ }^{1}$ Cathy Eng, ${ }^{4}$ Scott Kopetz, ${ }^{4}$ Michael J.

Overman, ${ }^{4}$ John M. Skibber, ${ }^{5}$ Miguel Rodriguez-Bigas, ${ }^{5}$ George J. Chang, ${ }^{5}$ Y. Nancy You, ${ }^{5}$

Brian K. Bednarski, ${ }^{5}$ Bruce D. Minsky, ${ }^{1}$ Marc E. Delclos, ${ }^{1}$ Eugene Koay, ${ }^{1}$ Sunil Krishnan, ${ }^{1}$

Christopher H. Crane, ${ }^{1,3}$ and Prajnan Das ${ }^{1}$

Department of ${ }^{1}$ Radiation Oncology, ${ }^{4}$ Gastrointestinal Medical Oncology and ${ }^{5}$ Surgical

Oncology, The University of Texas MD Anderson Cancer Center, Houston, TX, USA;

${ }^{2}$ Department of Radiation Oncology, Huntsman Cancer Institute, University of Utah, Salt Lake

City, UT, USA; ${ }^{3}$ Department of Radiation Oncology, Memorial Sloan Kettering Cancer Center,

New York, NY, USA

*Corresponding author: Randa Tao, MD, Department of Radiation Oncology, Huntsman

Cancer Hospital, University of Utah, 1950 Circle of Hope, Room 1570, Salt Lake City, UT,

84112, USA. Tel 1-801-213-6135; Fax 1-801-585-2666; E-mail: randa.tao@hci.utah.edu.

Pages: 16 (double-spaced text)

Tables: 3

Figures: 3

Running Title: Reirradiation for rectal cancer

Keywords: rectal cancer, recurrence, reirradiation, hyperfractionation

Presented in part as an oral presentation at the $58^{\text {th }}$ Annual Meeting of the American Society for Therapeutic Radiology and Oncology in Boston, MA. 


\section{Abstract}

\section{Background and Purpose:}

To evaluate outcomes and toxicity in patients treated with hyperfractionated pelvic reirradiation for recurrent rectal cancer.

Materials and Methods:

102 patients with recurrent rectal adenocarcinoma were treated with pelvic reirradiation with a hyperfractionated accelerated approach, consisting of 1.5 Gy twice daily fractions with a total dose of 30-45 Gy (median 39 Gy), with the most common total dose 39 Gy ( $n=90,88 \%$ ). The median dose of prior pelvic radiation therapy (RT) was 50.4 Gy (range: 25-63 Gy).

\section{Results:}

The median follow-up was 40 months for living patients (range, 3-150 months). The 3-year freedom from local progression (FFLP) rate was $40 \%$ and the 3-year overall survival (OS) rate was $39 \%$. Treatment with surgery was significantly associated with improved FFLP and OS, with 3 -year FFLP rate of $49 \%$ vs. $30 \%(P=.013)$, and 3 -year OS rate of $62 \%$ vs. $20 \%(P<.0001)$, compared to those without surgery. The actuarial 3-year rate of grade 3-4 late toxicity was 34\%; patients who underwent surgery had a significantly higher rate of grade 3-4 late toxicity compared to those without surgery $(54 \%$ vs. $16 \%, P=.001)$.

\section{Conclusions:}

This large, retrospective, single-institution study shows that hyperfractionated accelerated reirradiation was well tolerated. The rate of FFLP was promising, given that the study comprised heavily pre-treated patients with recurrences. Rates of FFLP and OS were particularly impressive in patients who underwent both reirradiation and surgery. 


\section{Introduction}

Patients with locally advanced rectal cancer have a risk of local recurrence despite multimodality treatment with surgery, radiation therapy (RT), and chemotherapy. Depending on the stage, the local recurrence risk is estimated to be 5-20\%.[1,2] While improvements in surgical techniques (total mesorectal excision) and the widespread use of preoperative chemoradiation therapy have led to a decreased risk of local recurrence, an increasing number of patients with recurrent rectal cancer have had a prior course of pelvic RT.[3-6]

The standard treatment for recurrent rectal cancer is not well established. Prior studies have shown that curative resection of the local recurrence is predictive of improved survival. Reirradiation could potentially increase the ability for patients to undergo curative surgery. Prior retrospective and phase I-II studies have shown reirradiation to be feasible and safe.[7-12]

At our institution, we have treated recurrent rectal cancer patients with a history of prior pelvic RT using a hyperfractionated accelerated reirradiation schedule. We reported our initial experience with this technique in 50 patients treated with reirradiation from 2001 to 2005 and showed that the treatment was well tolerated.[13] We performed the current study to further evaluate the outcomes and toxicity of this treatment in a much larger cohort of patients with longer follow-up.

\section{Materials and Methods}

\section{Patients}

This study was approved by the institutional review board. We identified 102 consecutive patients with a history of prior pelvic RT and with recurrent rectal adenocarcinoma, who were 
treated with reirradiation using a hyperfractionated accelerated approach between 2001 and 2012. The patient and treatment characteristics are summarized in Table 1.

\section{Radiation Treatment}

All patients were treated with a hyperfractionated accelerated approach consisting of 1.5 Gy

fractions delivered twice daily with a minimum 6 hour interval between fractions. Patients were excluded from reirradiation if the interval from the prior course of radiation therapy was deemed to be too short or if there was a large volume of small bowel that would be exposed to reirradiation. The majority of patients were treated with preoperative intent (64\%). Conditions that excluded patients from preoperative treatment included disease that would be grossly unresectable even with reirradiation, extensive metastatic disease, or a poor performance status that would preclude surgery. The median reirradiation dose was $39 \mathrm{~Gy}$ in 26 fractions (range, 30-45 Gy). The total reirradiation dose was determined, in part, by the duration of the retreatment interval. The most common reirradiation regimen of 39 Gy in 26 fractions $(n=90$, $88 \%$ ) was given if the retreatment interval was $\geq 1$ year while 30 Gy in 20 fractions $(n=8,8 \%)$ was utilized if the retreatment interval was <1 year. Two patients treated with 33 Gy and 36 Gy did not finish a planned treatment course of 39 Gy because of grade 3 acute toxicity, while two patients were treated with 42 Gy and 45 Gy at the treating physician's discretion.

The reirradiation field typically included the gross tumor volume (GTV) with a $2-3 \mathrm{~cm}$ block margin or a 1.5-2.5 cm planned target volume (PTV) margin. An example reirradiation treatment field is shown in the Supplemental Figure 1. The majority of patients were treated with a three-field technique and were positioned prone on a belly board device $(n=88,86 \%)$. The remaining patients were treated with 2 lateral fields $(n=5,5 \%)$, intensity-modulated radiation 
therapy (IMRT, $n=4,4 \%)$, or other techniques $(n=5,5 \%)$. In general, there was a low volume of small bowel in the retreatment field but in some cases with small bowel adjacent to the GTV, IMRT was used in an effort to minimize dose to the small bowel $(n=4)$. Treatment fields and blocks were designed to minimize dose to the small bowel and femoral heads, but, no specific dose constraints were used for treatment planning.

\section{Evaluation}

Patients were seen by the multidisciplinary team for follow-up and were assessed with radiographic imaging with a $\mathrm{CT}$ and/or MRI pelvis. Local progression (LP) was defined as any recurrence or disease progression in the pelvis found on imaging studies or by endoscopy. The medical records for patients were reviewed and information on disease status and toxicity were collected retrospectively. Acute and late toxicity were graded using the NCI Common Toxicity Criteria for Adverse Events (CTCAE) version 4 guidelines.

\section{Statistical Analysis}

The primary outcomes assessed were freedom from local progression (FFLP), overall survival (OS), and rates of late toxicity. These outcomes were estimated by the Kaplan-Meier method using the date of reirradiation completion as the start time. The log rank test was used to identify variables that were associated with differences in outcomes. Univariate analysis was also done with the Cox regression model for continuous variables. The Cox proportional hazards model was performed for multivariate analysis for FFLP and OS. The number of variables included in the model was about 1 per 10 events using the variables with $P$ values $<.15$ on univariate analysis.

\section{Results}




\section{Treatment Characteristics}

The median dose of prior pelvic RT was $50.4 \mathrm{~Gy}$ (range: $25-63 \mathrm{~Gy}$ ) and the median cumulative RT dose was 89.4 Gy (range: 55-102 Gy without intraoperative radiation therapy [IORT] and 55104.5 Gy with IORT). The median time from prior RT to reirradiation was 30 months (range: 5789 months).

Ninety-three patients $(91 \%)$ received concurrent chemotherapy with reirradiation, including capecitabine in 89 patients (87\%), 5-fluorouracil (5-FU) in 2 patients, 5-FU and oxaliplatin in 1 patient, and cisplatin and irinotecan in 1 patient.

In total, $66 \%(43 / 65)$ of the patients treated with a preoperative intent ultimately underwent surgical resection. The reasons that patients did not undergo surgery included evidence of local and/or distant disease progression $(n=15)$, pelvic disease that was determined to still be unresectable after reirradiation $(n=3)$, patient refusal $(n=3)$, and a decline in performance status $(\mathrm{n}=1)$. A total of forty-six patients $(45 \%)$ were treated with surgical resection; 43 patients underwent surgery after reirradiation while 3 patients had surgery prior to reirradiation. The type of surgeries included pelvic exenteration in 19 patients, abdominal perineal resection (APR) with multivisceral resection in 7 patients, APR alone in 5 patients, tumor resection in 6 patients, low anterior resection (LAR) in 2 patients, multivisceral resection in 3 patients, and other surgery types in the remaining 4 patients.

Of the patients undergoing surgery, 31 (67\%) had an R0 resection.

Twenty-two patients who underwent surgery also received IORT to a dose of $7.5 \mathrm{~Gy}$ $(\mathrm{n}=1), 10 \mathrm{~Gy}(\mathrm{n}=11), 12.5 \mathrm{~Gy}(\mathrm{n}=6)$, and $15 \mathrm{~Gy}(\mathrm{n}=4)$. IORT was delivered using high dose rate 
(HDR) brachytherapy, using an iridium-192 source, with the dose prescribed at $1 \mathrm{~cm}$ from the source.

\section{Pelvic Control}

The median follow-up was 28 months (range, 1-150 months) for all patients and 40 months for patients alive at the time of analysis (range, 3-150 months). A total of 38 patients (37\%) developed evidence of LP at a median time of 25 months. The FFLP rate at 2 and 3 years from the completion of reirradiation was $51 \%$ and $40 \%$, respectively (Supplemental Fig. 2A). Treatment with surgery was significantly associated with improved FFLP, with a 3-year FFLP rate of $49 \%$ for patients who underwent surgery vs. $30 \%$ for those who did not $(P=.013$, Fig. 1A). The median time to LP was 27 months for patients who underwent surgery vs. 16 months for those who did not.

The retreatment interval was not found to be significantly associated with the rate of FFLP $(P=.11)$. The retreatment radiation dose $(P=.07)$ and the cumulative dose $(P=.17)$ were not found to be significantly associated with the rate of FFLP.

Surgery remained a significant predictor of FFLP (hazard ratio $[\mathrm{HR}]=0.42,95 \%$ confidence interval $[\mathrm{CI}]=0.22-0.81, P=.009)$ on multivariate analysis. The multivariate analysis included the retreatment interval and retreatment radiation dose, which were not found to be significantly associated with FFLP.

Among the 46 patients who underwent surgery, the type of surgery performed $(P=.94)$ and the surgical margins $(P=.56)$ were not associated with significant differences in FFLP. This is likely due to the fact that the majority of patients had an R0 resection. There was also no significant association between treatment with IORT and FFLP $(P=.45)$. We also examined the 
surgical pathology for these patients and found that 6 patients $(14 \%)$ had a pathologic complete response at surgery after treatment with reirradiation. For the patients without a pathologic complete response, tumor viability was quantified in the surgical specimen for 17 patients. The percent of viable tumor ranged from $1-95 \%$ with a median of $40 \%$ (interquartile range $=12.5$ $60 \%)$.

Symptom Control

For the patients treated with palliative intent who had identifiable symptoms, an estimated $70 \%$ (21/30) experienced palliation of their symptoms (Table 2). These symptoms included pain, bleeding, and urinary obstruction (hydroureter and hydronephrosis). The median duration of symptom relief after reirradiation was 10 months (range, 1-24). Four patients experienced permanent relief of their symptoms.

\section{Overall Survival}

At the time of analysis, a total of 80 patients had died (80\%). The median OS was 30 months and the 2- and 3-year OS rates were 61\% and 39\%, respectively (Supplemental Fig. 2B). Treatment with surgery was significantly associated with improved OS. The 3-year OS rate was $63 \%$ for patients who were able to undergo surgery compared to $20 \%$ for those who were not able to receive surgical resection $(P<.0001$, Fig. 1B). The median OS for patients who received surgery was 47 months compared to 17 months for those who did not. Patients with metastatic disease had a worse median OS of 32 months compared to 47 months for patients without metastatic disease but this difference was not significant $(P=.09)$.

On multivariate analysis, surgery remained a strong predictor of OS $(\mathrm{HR}=0.34,95 \%$ $\mathrm{CI}=0.20-0.55, P<.0001)$ while the other variables in the multivariate model were not significant, 
including metastatic disease status $(P=.24)$, the retreatment RT dose $(P=.14)$, and carcinoembryonic antigen level $(P=.12)$.

\section{Toxicity}

The actuarial 3-year rate of grade 3-4 late toxicity was 34\%; patients who underwent surgery had a significantly higher rate of grade 3-4 late toxicity compared to those who did not undergo surgery (54\% vs. $16 \%, P=.001$, Fig. 1C). Patients treated with a prior RT dose of $\geq 54$ Gy (n=14) had a trend towards a higher rate of grade 3-4 late toxicity compared to those treated with prior dose of $<54$ Gy $(57 \%$ vs. $26 \%, P=.053)$. A shorter retreatment interval of $\leq 24$ months was also associated with a trend towards a higher rate of late toxicity (43\% vs. $16 \%, P=.058)$.

The retreatment RT dose was not associated with a significant difference in the rate of late toxicity $(P=.51)$. The cumulative external beam dose was not associated with a higher rate of late toxicity $(P=.11)$. Among the surgery patients, treatment with IORT was not associated with increased toxicity.

In a multivariate model including treatment with surgery, cumulative external beam dose, and treatment with a prior dose of $\geq 54$ Gy as covariates, surgery remained a significant predictor of grade 3-4 late toxicity $(\mathrm{HR}=2.96,95 \% \mathrm{CI}=1.30-7.32, P=.012)$ while the other factors were not significant. The types of toxicity experienced by patients also differed between the groups.

Grade 3 late toxicities in the surgery group included wound complications with infection and/or a non-healing wound $(n=8)$, pelvic abscess $(n=8)$, small bowel obstruction $(n=3)$, urinary obstruction/hydronephrosis $(n=2)$, renal failure $(n=2)$, ureteral stricture $(n=4)$, anastomotic stricture $(n=2)$, ureteral anastomotic leak $(n=1)$, cystitis $(n=2)$, proctitis $(n=3)$, diarrhea and dehydration $(n=1)$, fistula formation $(n=1)$, pain $(n=1)$, and anemia $(n=1)$, with some patients 
having multiple late toxicities. Grade 3 toxicities in the non-surgery group included small bowel obstruction $(n=3)$, urinary obstruction/hydronephrosis $(n=2)$, pain $(n=1)$, diarrhea and dehydration $(n=1)$, and fistula formation $(n=1)$.

\section{Discussion}

In a large retrospective analysis of reirradiation for patients with rectal cancer, we found promising outcomes in this cohort of heavily pre-treated patients with recurrent disease. The outcomes were especially encouraging in patients that underwent both reirradiation and surgery, with 3-year FFLP and OS rates of $49 \%$ and $62 \%$, respectively. However, even patients who were unable to undergo surgery still achieved a median duration of FFLP of 16 months. In this analysis that included a majority of patients who were followed until death, the rate of late toxicity was acceptable, and was particularly low for patients who did not undergo surgery. Furthermore, $14 \%$ of patients treated with reirradiation and surgery were found to have pathologic complete response, with the rate similar to that reported in randomized studies on preoperative chemoradiation for primary rectal cancers using standard fractionation. $[5,6,14$, $15]$

As preoperative chemoradiation therapy $[5,16]$ and short course $\mathrm{RT}[17,18]$ have been established as standard of care treatments for rectal cancer, patients who experience disease recurrence often have had a prior course of pelvic RT. This presents additional challenges in the management of these patients. Historically, reirradiation was not offered due to the concern of unacceptable toxicity. The use of a hyperfractionated approach to reirradiation is one strategy employed to decrease the risk of late effects, taking advantage of the radiobiologic differences between the proliferating tumor cells and the late-responding normal tissues.[19] 
Hyperfractionated schedules have thus been used in the majority of studies on reirradiation for rectal cancer, which are summarized in Supplemental Table 1.

Valentini and colleagues performed the landmark phase II multicenter prospective study of reirradiation, enrolling 59 patients with recurrent rectal cancer whose disease was limited to the pelvis.[12] In this study, patients were treated with 1.2 Gy twice daily fractions to a dose of 40.8 Gy with concurrent continuous infusion of 5-FU. Thirty-nine patients (66\%) were able to undergo surgical resection with 21 patients (54\%) achieving an R0 resection. Similar to our findings, 5 patients (13\% of those undergoing surgery) experienced a pathologic complete response. They reported a 3-year local control rate of $47 \%$, which is also similar to our results. A large prospective study on reirradiation for rectal cancer was reported by Sun and colleagues in 2012.[20] They included 72 patients who were considered to have unresectable locally recurrent rectal cancer treated with 1.2 Gy twice daily to a total dose of 30-36 Gy with concurrent capecitabine. Eighteen patients (25\%) underwent resection including 16 that had an $\mathrm{R} 0$ resection. The remaining patients were treated to a higher total dose of 51.6-56.4 Gy. They reported a 3-year progression-free survival rate of $31 \%$ and OS rate of $45 \%$.

In addition to our prior study, several other retrospective studies have been published on reirradiation using both hyperfractionated and conventional fractionation (Supplemental Table 1).[7-10, 21-24] Surgical resection was performed in $20-99 \%$ of patients, which is an expectedly wide range; our series falls in the middle of this range with $45 \%$ of patients undergoing surgery. Survival outcomes in prior series were also improved in patients who were able to undergo surgery; the median OS was 39-60 months compared with 12-16 months for patients who did not undergo surgery. 
The toxicity rates described in the literature for reirradiation are generally acceptable, with acute grade 3-4 toxicity rates of $4-20 \%$ and late toxicity rates of $12-39 \% .[9,12,13,22,24]$ In our series, patients who underwent surgery had a significantly higher rate of late toxicity. The crude rate was approximately 3 times higher in the patients who underwent surgery and the HR for surgery on multivariate analysis was also close to 3 . The magnitude of this increased toxicity and the fact these patients were treated with complex surgeries, often with multi-organ resection, suggest that much of the late toxicity may be attributed to surgery. The low rates of late toxicity in the patients treated without surgery are promising and may further support the use of reirradiation.

Our institution's overall management approach for locally recurrent rectal cancer was recently reported [25]. Over a 24 year period, 229 patients underwent multimodal curative therapy, including surgery, for locally recurrent rectal cancer. Of these patients, $73 \%$ received preoperative radiotherapy and/or chemotherapy, $60 \%$ received preoperative radiation therapy, and $38 \%$ received postoperative chemotherapy. Among patients treated with preoperative radiation therapy, 59\% had received no prior radiation therapy and were treated with conventional fractionation (50.4 Gy in 28 daily fractions), while $41 \%$ had received prior pelvic radiotherapy, and underwent hyperfractionated accelerated reirradiation, as described in the current study. Thus, reirradiation was an important component of management in a large proportion of patients with locally recurrent rectal cancer. This proportion is likely to increase over time, given the increasing adoption of preoperative radiation therapy for rectal cancer.

As with all retrospective studies, our study has inherent limitations. Treatment-related toxicity may be underestimated as some toxicity may not have been fully documented in the medical record. Furthermore, determination of symptom relief was subjective. Another 
limitation is that it is difficult to determine how much reirradiation contributed to the outcomes for these patients given that they received multimodality treatment. Some patients underwent surgery while others were considered inoperable or refused surgery, which reflects the heterogeneity of patients with recurrent disease. A strength of this study is that a relatively uniform approach to reirradiation was used, with 1.5 Gy twice daily fractions in all patients, and a total dose of 39 Gy in $88 \%$ of patients. Further, this study has robust follow-up to capture late events as the majority of the patients were followed until death. With 102 patients, our study adds to the literature as one of the largest cohorts of rectal cancer patients treated with reirradiation. The data presented in this study may be the basis of future prospective studies that can further evaluate the role of reirradiation and its effect on pelvic control, or whether radiosensitizers can be used to improve the efficacy of reirradiation.

In summary, our retrospective data show that hyperfractionated accelerated reirradiation was well tolerated. The rate of FFLP was promising, given that the study comprised heavily pretreated patients with recurrences. Rates of FFLP and OS were particularly encouraging in patients who underwent both reirradiation and surgery. Prospective trials are warranted to further evaluate ways to improve outcomes in this population. 


\section{Conflict of Interest Statement}

The authors declare no conflicts of interest related to this work. Dr. Sunil Krishnan reports grants from the National Institutes of Health, US Department of Defense, MD Anderson Cancer Center, Focused Ultrasound Surgery Foundation, Shell Oil, Malaysian Palm Oil Board, Dunn Foundation, and Elekta, grants from Celgene and Genentech, and royalties from Taylor and Francis outside the submitted work. In addition, Dr. Krishnan has a patent pending for radiosensitization with nanoparticles licensed to self (from MD Anderson Cancer Center).

\section{Figure Legends}

Supplemental Fig 1. Example reirradiation treatment fields with a posterior-anterior (PA) (A) and lateral (B) field. The gross tumor volume (GTV) is contoured in red. This patient was treated prone on a belly board device with a 3-field technique to 39 Gy in 261.5 Gy twice daily fractions.

Supplemental Fig 2. Kaplan-Meier estimates of freedom from local progression (A) and overall survival (B).

Fig. 1. Effect of surgery on freedom from local progression (A), overall survival (B), and the rate of grade 3-4 late toxicity (C).

\section{References}

[1] Gunderson LL, Sargent DJ, Tepper JE, Wolmark N, O'Connell MJ, Begovic M, et al. Impact of T and $\mathrm{N}$ stage and treatment on survival and relapse in adjuvant rectal cancer: a pooled analysis. Journal of clinical oncology : official journal of the American Society of Clinical Oncology. 2004;22:1785-96.

[2] Das P, Skibber JM, Rodriguez-Bigas MA, Feig BW, Chang GJ, Hoff PM, et al. Clinical and pathologic predictors of locoregional recurrence, distant metastasis, and overall survival in patients treated with chemoradiation and mesorectal excision for rectal cancer. American journal of clinical oncology. 2006;29:219-24.

[3] Heald RJ, Ryall RD. Recurrence and survival after total mesorectal excision for rectal cancer. Lancet. 1986;1:1479-82.

[4] Braendengen M, Tveit KM, Berglund A, Birkemeyer E, Frykholm G, Pahlman L, et al. Randomized phase III study comparing preoperative radiotherapy with chemoradiotherapy in 
nonresectable rectal cancer. Journal of clinical oncology : official journal of the American Society of Clinical Oncology. 2008;26:3687-94.

[5] Sauer R, Becker H, Hohenberger W, Rodel C, Wittekind C, Fietkau R, et al. Preoperative versus postoperative chemoradiotherapy for rectal cancer. The New England journal of medicine. 2004;351:1731-40.

[6] Gerard JP, Conroy T, Bonnetain F, Bouche O, Chapet O, Closon-Dejardin MT, et al. Preoperative radiotherapy with or without concurrent fluorouracil and leucovorin in T3-4 rectal cancers: results of FFCD 9203. Journal of clinical oncology : official journal of the American Society of Clinical Oncology. 2006;24:4620-5.

[7] Lingareddy V, Ahmad NR, Mohiuddin M. Palliative reirradiation for recurrent rectal cancer. International journal of radiation oncology, biology, physics. 1997;38:785-90.

[8] Mohiuddin M, Lingareddy V, Rakinic J, Marks G. Reirradiation for rectal cancer and surgical resection after ultra high doses. International journal of radiation oncology, biology, physics. 1993;27:1159-63.

[9] Mohiuddin M, Marks G, Marks J. Long-term results of reirradiation for patients with recurrent rectal carcinoma. Cancer. 2002;95:1144-50.

[10] Mohiuddin M, Marks GM, Lingareddy V, Marks J. Curative surgical resection following reirradiation for recurrent rectal cancer. International journal of radiation oncology, biology, physics. 1997;39:643-9.

[11] Valentini V, Morganti AG, De Franco A, Coco C, Ratto C, Battista Doglietto G, et al. Chemoradiation with or without intraoperative radiation therapy in patients with locally recurrent rectal carcinoma: prognostic factors and long term outcome. Cancer. 1999;86:261224.

[12] Valentini V, Morganti AG, Gambacorta MA, Mohiuddin M, Doglietto GB, Coco C, et al. Preoperative hyperfractionated chemoradiation for locally recurrent rectal cancer in patients previously irradiated to the pelvis: A multicentric phase II study. International journal of radiation oncology, biology, physics. 2006;64:1129-39.

[13] Das P, Delclos ME, Skibber JM, Rodriguez-Bigas MA, Feig BW, Chang GJ, et al. Hyperfractionated accelerated radiotherapy for rectal cancer in patients with prior pelvic irradiation. International journal of radiation oncology, biology, physics. 2010;77:60-5. [14] Bosset JF, Calais G, Mineur L, Maingon P, Radosevic-Jelic L, Daban A, et al. Enhanced tumorocidal effect of chemotherapy with preoperative radiotherapy for rectal cancer: preliminary results--EORTC 22921. Journal of clinical oncology : official journal of the American Society of Clinical Oncology. 2005;23:5620-7.

[15] Roh MS, Colangelo LH, O'Connell MJ, Yothers G, Deutsch M, Allegra CJ, et al. Preoperative multimodality therapy improves disease-free survival in patients with carcinoma of the rectum: NSABP R-03. Journal of clinical oncology : official journal of the American Society of Clinical Oncology. 2009;27:5124-30.

[16] Sauer R, Liersch T, Merkel S, Fietkau R, Hohenberger W, Hess C, et al. Preoperative versus postoperative chemoradiotherapy for locally advanced rectal cancer: results of the German CAO/ARO/AIO-94 randomized phase III trial after a median follow-up of 11 years. Journal of clinical oncology : official journal of the American Society of Clinical Oncology. 2012;30:1926-33. 
[17] Improved survival with preoperative radiotherapy in resectable rectal cancer. Swedish Rectal Cancer Trial. The New England journal of medicine. 1997;336:980-7.

[18] Ngan SY, Burmeister B, Fisher RJ, Solomon M, Goldstein D, Joseph D, et al. Randomized trial of short-course radiotherapy versus long-course chemoradiation comparing rates of local recurrence in patients with T3 rectal cancer: Trans-Tasman Radiation Oncology Group trial 01.04. Journal of clinical oncology : official journal of the American Society of Clinical Oncology. 2012;30:3827-33.

[19] Thames HD, Jr., Withers HR, Peters LJ, Fletcher GH. Changes in early and late radiation responses with altered dose fractionation: implications for dose-survival relationships. International journal of radiation oncology, biology, physics. 1982;8:219-26.

[20] Sun DS, Zhang JD, Li L, Dai Y, Yu JM, Shao ZY. Accelerated hyperfractionation field-involved re-irradiation combined with concurrent capecitabine chemotherapy for locally recurrent and irresectable rectal cancer. The British journal of radiology. 2012;85:259-64.

[21] Koom WS, Choi Y, Shim SJ, Cha J, Seong J, Kim NK, et al. Reirradiation to the pelvis for recurrent rectal cancer. Journal of surgical oncology. 2012;105:637-42.

[22] Ng MK, Leong T, Heriot AG, Ngan SY. Once-daily reirradiation for rectal cancer in patients who have received previous pelvic radiotherapy. Journal of medical imaging and radiation oncology. 2013;57:512-8.

[23] Haddock MG, Miller RC, Nelson H, Pemberton JH, Dozois EJ, Alberts SR, et al. Combined modality therapy including intraoperative electron irradiation for locally recurrent colorectal cancer. International journal of radiation oncology, biology, physics. 2011;79:143-50.

[24] Bosman SJ, Holman FA, Nieuwenhuijzen GA, Martijn H, Creemers GJ, Rutten HJ. Feasibility of reirradiation in the treatment of locally recurrent rectal cancer. Br J Surg. 2014;101:1280-9. [25] You YN, Skibber JM, Hu CY, Crane CH, Das P, Kopetz ES, et al. Impact of multimodal therapy in locally recurrent rectal cancer. Br J Surg. 2016. 
[Figure 1]

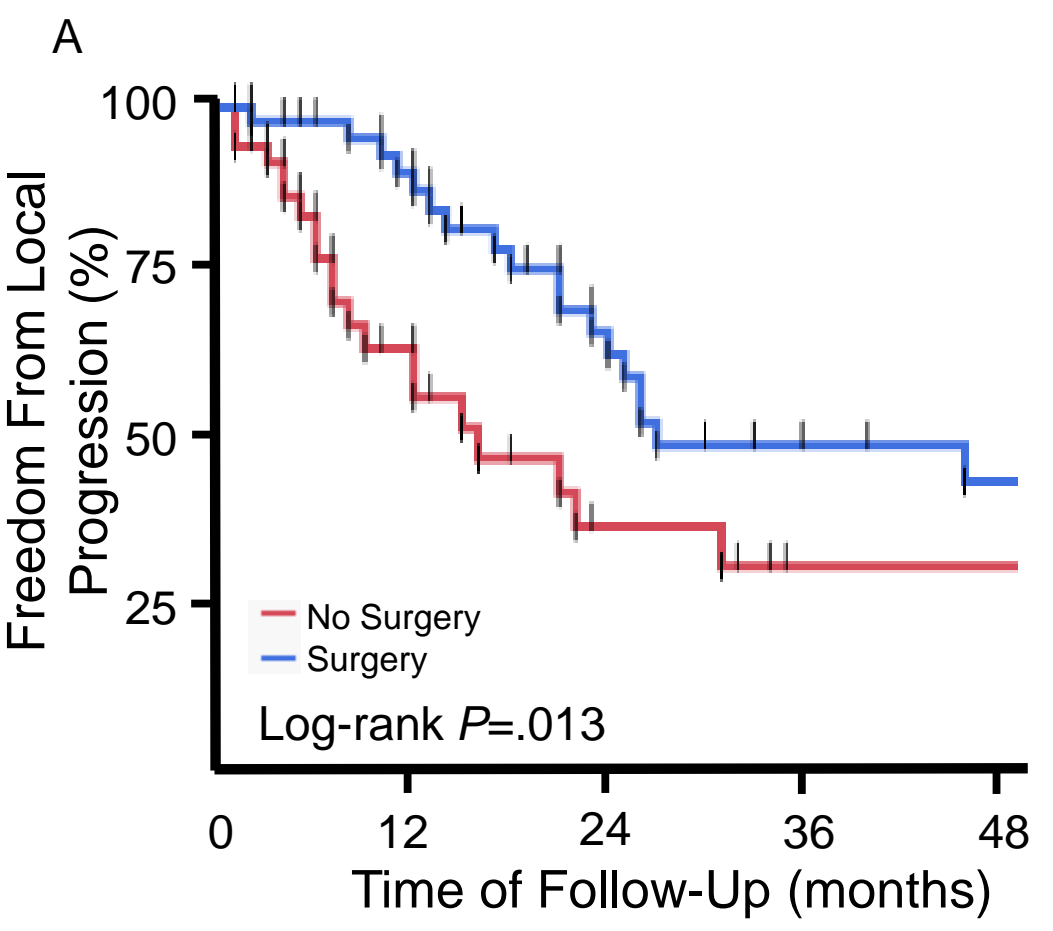

- No Surgery 56

- Surgery 46
17

33
7

19
3

12
B

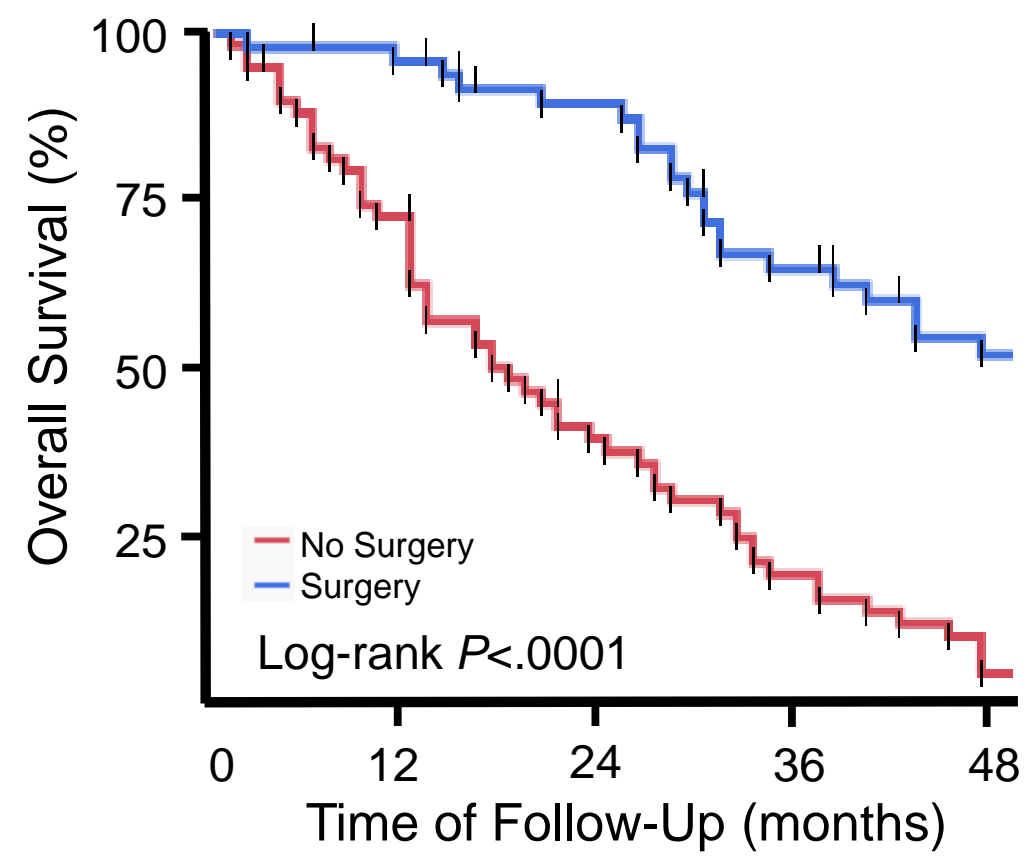

$\begin{array}{ll}\text { - No Surgery } & 56 \\ \text { - Surgery } & 46\end{array}$

39

21

38 5
11

26 
[Figure 1]

C

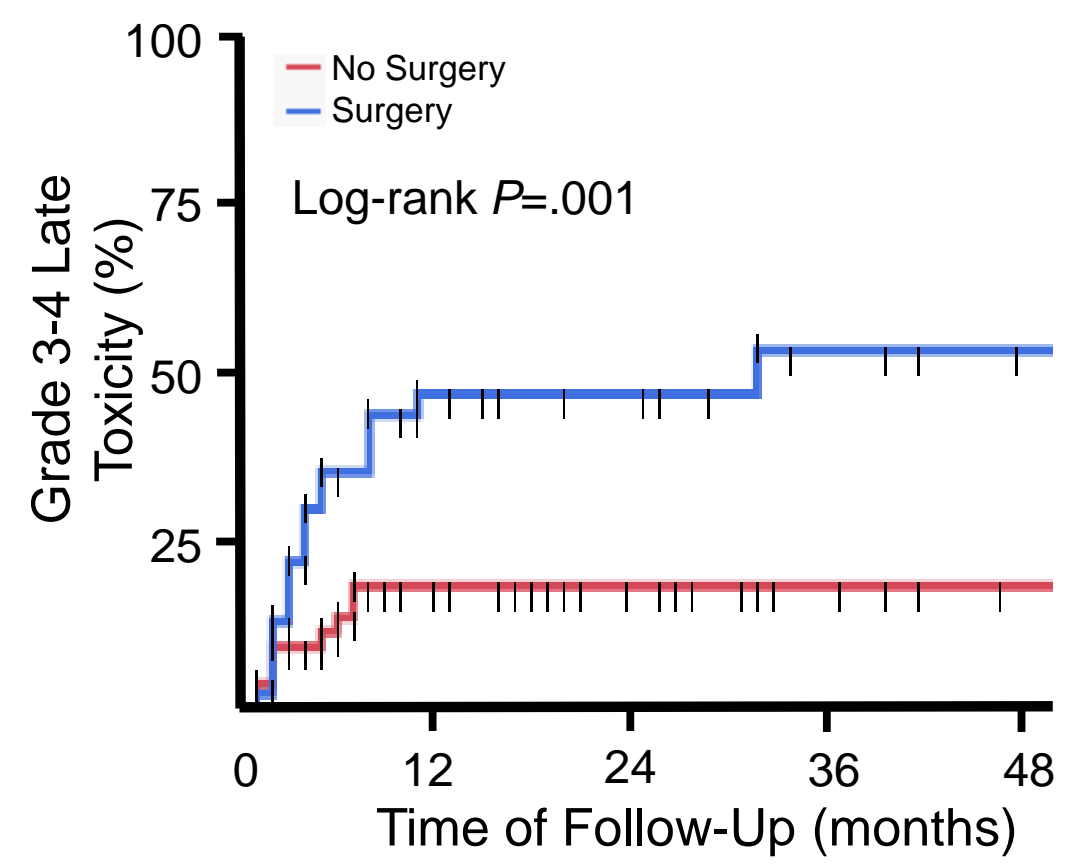

- No Surgery 56

30

16

10

- Surgery

46

19

11

4
4 
Table 1. Patient and Treatment Characteristics

\begin{tabular}{|c|c|c|}
\hline & $\begin{array}{l}\text { Median or } \\
\text { Number }\end{array}$ & $\begin{array}{l}\text { Range or } \\
\% \text { of total }\end{array}$ \\
\hline \multicolumn{3}{|l|}{ Number of patients } \\
\hline Men & 39 & $38 \%$ \\
\hline Women & 63 & $62 \%$ \\
\hline Median age, years & 58 & $35-77$ \\
\hline \multicolumn{3}{|l|}{ Race } \\
\hline White & 68 & $67 \%$ \\
\hline Hispanic & 21 & $20 \%$ \\
\hline Black & 12 & $12 \%$ \\
\hline Asian & 1 & $1 \%$ \\
\hline $\mathrm{CEA}, \mu \mathrm{g} / \mathrm{L}$ & 5.8 & $0.5-3469$ \\
\hline Median prior RT dose & $50.4 \mathrm{~Gy}$ & 25-63 Gy \\
\hline Median retreatment interval & $30 \mathrm{mo}$. & 5-789 mo. \\
\hline \multicolumn{3}{|l|}{ Retreatment intent } \\
\hline Preoperative & 65 & $64 \%$ \\
\hline Palliative & 34 & $33 \%$ \\
\hline Postoperative & 3 & $3 \%$ \\
\hline \multicolumn{3}{|l|}{ Retreatment dose } \\
\hline 39 Gy in $26 \mathrm{fx}$ & 90 & $88 \%$ \\
\hline 30 Gy in $20 \mathrm{fx}$ & 8 & $8 \%$ \\
\hline 33 Gy in $22 \mathrm{fx}$ & 1 & $1 \%$ \\
\hline 36 Gy in $24 \mathrm{fx}$ & 1 & $1 \%$ \\
\hline $42 \mathrm{~Gy}$ in $28 \mathrm{fx}$ & 1 & $1 \%$ \\
\hline 45 Gy in $30 \mathrm{fx}$ & 1 & $1 \%$ \\
\hline Median cumulative RT dose & 89.4 Gy & 55-102 Gy (not incl. IORT) \\
\hline & & 55-104.5 Gy (incl. IORT) \\
\hline \multicolumn{3}{|l|}{ Concurrent chemotherapy } \\
\hline Yes & 93 & $91 \%$ \\
\hline No & 9 & $9 \%$ \\
\hline \multicolumn{3}{|l|}{ Surgical resection } \\
\hline Yes & 46 & $45 \%$ \\
\hline No & 56 & $55 \%$ \\
\hline
\end{tabular}

Abbreviations: CEA, carcinoembryonic antigen; RT, radiation therapy; mo., months; Gy, Gray; fx, fractions; IORT, intraoperative radiation therapy; incl., including 
Table 2. Palliation of Symptoms

\begin{tabular}{llll}
\hline Symptom & $\mathrm{N}^{*}$ & $\begin{array}{l}\text { Proportion of } \\
\text { Patients with } \\
\text { Symptom Relief }\end{array}$ & $\begin{array}{l}\text { Duration of } \\
\text { Symptom Relief } \\
\text { (Median) }\end{array}$ \\
\hline Pain & 19 & $79 \%$ & 9 months \\
Bleeding & 3 & $100 \%$ & 21 months \\
$\begin{array}{l}\text { Urinary } \\
\text { obstruction }\end{array}$ & 8 & $63 \%$ & 8 months \\
\hline
\end{tabular}

Abbreviation: N, number of patients with each symptom

*Some patients had multiple symptoms 